CORRECTION

https://doi.org/10.1038/s41586-018-0815-6

\title{
Author Correction: Autophagy maintains tumour growth through circulating arginine
}

Laura Poillet-Perez, Xiaoqi Xie, Le Zhan, Yang Yang, Daniel W. Sharp, Zhixian Sherrie Hu, Xiaoyang Su,

Anurag Maganti, Cherry Jiang, Wenyun Lu, Haiyan Zheng,

Marcus W. Bosenberg, Janice M. Mehnert, Jessie Yanxiang Guo, Edmund Lattime, Joshua D. Rabinowitz \& Eileen White

Correction to: Nature https://www.nature.com/articles/s41586-0180697-7, published online 14 November 2018.

In the Abstract of this Letter, 'released' should have been 'regulated' in the sentence: 'Deletion of Atg5 in the host similarly regulated circulating arginine and suppressed tumorigenesis, which demonstrates that this phenotype is specific to autophagy function rather than to deletion of Atg7.' This has been corrected online. 